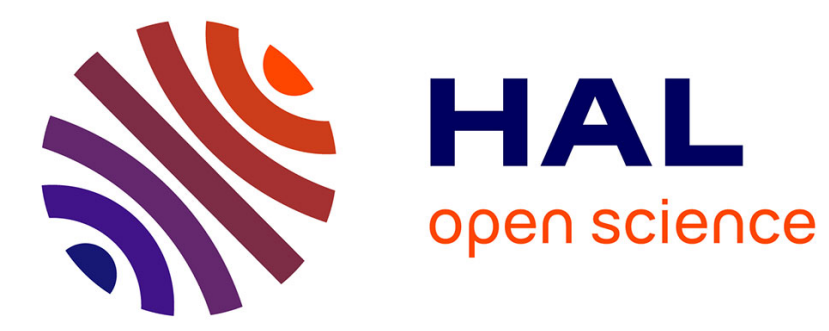

\title{
Potentialities and limitations of hologram multiplexing by using the phase-encoding technique
} Cornélia Denz, Gilles Pauliat, Gérald Roosen, Theo Tschudi

\section{To cite this version:}

Cornélia Denz, Gilles Pauliat, Gérald Roosen, Theo Tschudi. Potentialities and limitations of hologram multiplexing by using the phase-encoding technique. Applied optics, 1992, 31 (26), pp.5700-5705. 10.1364/AO.31.005700 . hal-00875938

\section{HAL Id: hal-00875938 \\ https://hal-iogs.archives-ouvertes.fr/hal-00875938}

Submitted on 23 Oct 2013

HAL is a multi-disciplinary open access archive for the deposit and dissemination of scientific research documents, whether they are published or not. The documents may come from teaching and research institutions in France or abroad, or from public or private research centers.
L'archive ouverte pluridisciplinaire HAL, est destinée au dépôt et à la diffusion de documents scientifiques de niveau recherche, publiés ou non, émanant des établissements d'enseignement et de recherche français ou étrangers, des laboratoires publics ou privés. 


\title{
Potentialities and limitations of hologram multiplexing by using the phase-encoding technique
}

\author{
C. Denz, G. Pauliat, G. Roosen, and T. Tschudi
}

\begin{abstract}
The advantages and limitations of data storage in holographic materials by implementing a pure phase-encoding method of the reference beam are studied. We show that if deterministic orthogonal binary phase addresses are used, such a system is theoretically able to store as many images as the usual angular multiplexing method. However, we demonstrate that imperfections of available optical components generate optical noise and limit the storage capacity. We propose an improved recording technique to overcome some of these limitations.
\end{abstract}

\section{Introduction}

Multiplexing a large number of gratings in a holographic medium in such a way that all images stored can be addressed independently is important for a wide range of forthcoming applications. ${ }^{1}$ For instance, at an elementary level, holographic gratings can be used to implement interconnection links between individual processing elements of two distinct places in point-to-point interconnection structures. ${ }^{2}$ Reconfigurable holograms are also important for dynamic optical memories because of the parallelism of optics and because the high storage capacity of these recording media allows for a large number of independently retrievable images to be set up by multiplexing multiple gratings..$^{3-5}$

There are two different ways $^{6}$ to perform this multiplexing. In the first of them, each grating is allocated some volume (or area for thin media) in the holographic medium that is not shared by other gratings. In the second configuration, all gratings share the same volume-area of the material. To avoid cross talk between reconstructed images, we

When this work was performed C. Denz, G. Pauliat, and G. Roosen were with the Institut d'Optique Théorique et Appliquée, Unite Associée au Centre National de la Recherche Scientifique, Centre Scientifique d'Orsay, B.P. 147.91403 Orsay Cedex, France. T. Tschudi was with the Institut für Agnewandte Physik, Technische Hochschule Darmstadt, Hochschulstrasse 6, W-6100 Darmstadt, Germany. C. Denz's permanent address is with the Institut für Angewandte Physik, Technische Hochschule Darmstadt, Hochschulstrasse 6, W-1600 Darmstadt, Germany.

Received 17 June 1991.

0003-6935/92/265700-06\$05.00/0.

- 1992 Optical Society of America. can either rely on the Bragg selectivity of thick media to reconstruct one image at a time, or we can reconstruct the images so that the unwanted reconstructed images do not overlap the desired reconstructed image. 6,7

Our work has concentrated on a configuration in which all gratings share the same volume in the recording medium. In this configuration, in order to retrieve the images without ambiguity, each of these images must possess its own address. Most often, the address of the recorded image is represented by the incident angle of the reference beam used during the recording. This angular reference-beam multiplexing has become a widely spread method used in different schemes for associative memories and highcapacity data storage. ${ }^{8-11}$ In order to change this reference-beam incident angle acousto-optic deflectors were proposed, but the necessity of using two acousto-optic devices ${ }^{12}$ to compensate for the Doppler frequency shift increases the complexity of the optical setup. Electro-optic translation devices were also proposed. ${ }^{12}$ Mechanical changes of the referencebeam angle are widely used..$^{3-5}$

Alternatively, the use of an intensity spatial light modulator to define the various incident directions was demonstrated. ${ }^{13}$ Nevertheless these two last methods still suffer from some disadvantages: mechanically changing the incident angle of the reference beam requires a high reliability in the positioning and is therefore inherently slow, whereas amplitude modulation is energy consuming. Therefore the realization of a fast addressing system with low cross talk and energy efficient retrieval of the images still remains a problem.

For these reasons, we discuss here the advantages 
of the phase-encoding technique in which the addresses of the images are represented by the phase pattern of the reference beam.

Phase encoding has been proposed in different contexts, such as a supplementary coding address superposed on angularly multiplexed references for obtaining a larger signal-to-noise ratio ${ }^{14}$ or as a method for obtaining variable and modifiable interconnections weights by means of a double-exposure technique of phase-shifted holograms. ${ }^{15}$ Although pure phase-addressing multiplexing is promising, there have been only a few attempts to use pure phase encoding as a means of data multiplexing in photosensitive media. Random phase encoding of the reference beam by a diffuser was first proposed for multiplexing a number of point-source objects for data storage in the same area of a thin holographic plate. ${ }^{16}$ Deterministic phase encoding by a spatial light modulator was also demonstrated. In some of these attempts, an array of plane waves was encoded with a binary phase vector and registered in different areas of a thin holographic medium. ${ }^{17,18}$ Sinusoidally phase-modulated waves were also employed ${ }^{19}$ to record images in thick holographic media.

In contrast to these investigations, we discuss hereafter the advantages and limitations of a new deterministic phase-encoding method proposed recently, ${ }^{20,21}$ which minimizes the space-bandwidth product of the spatial phase modulator, e.g., the number of pixels of this binary modulator must be equal to only the number of images to be recorded. In this technique, each image is stored with $N$ pure and deterministic binary reference beams. Each set of adjustable phases for these reference beams represents the address of one of the $N$ stored images and is orthogonal with all other phase addresses. All reference beams overlap the image in the recording volume and interact independently with this image. Noiseless reconstruction appears because the reconstructions of the undesired images interfere destructively to produce zero intensity. Moreover, phaseonly addressing is naturally light efficient and provides simple and quick generated reference-beam patterns, thus permitting an easy image retrieval procedure with a high storage capacity.

Afterwards, in a mathematical approach, we first calculate the set of orthogonal phase addresses required for obtaining noiseless reconstruction in an ideal system and we derive the storage capacity of that system. Next we discuss the limiting factors of realistic systems which arise from (a) the imperfections of the recording medium and (b) the imperfections of the phase-coding element. Then we propose an improved encoding scheme in order to overcome some limitations that are due to the phase encoder.

\section{Principle of Phase Encoding}

Because the diffraction efficiency of the holographic media depends on the fringe spacing of the interference pattern, we derive the following equations that approximate all the incident optical waves (image and reference beams) by plane waves. The phase-encoding technique is, of course, also efficient for nonplane waves.

We thus describe the $m$ th image beam by the complex amplitude $\mathscr{A}_{m}=0.5\left[A_{m} \exp (j \mathbf{k} \times \mathbf{r})+\right.$ c.c. $]$. The information of this image is spatially encoded on the amplitude $A_{m}$ while all vectors $\mathbf{k}$ are identical for all images and $j=\sqrt{-1}$ and $\mathbf{r}$ is a coordinate vector. During recording, this wave simultaneously interferes with the set of $N$ phase-modulated reference beams inside the holographic medium. These different reference beams can be generated by using, e.g., a computer-generated hologram or a microlens array to achieve separated pixels and a phase encoder based on liquid crystals. The complex amplitude of the $n$th reference beam is expressed by $\mathscr{P}_{n}=$ $0.5\left[P_{n} \exp \left(j \varphi_{n}{ }^{m}\right) \exp \left(j \mathbf{k}_{n} \times \mathbf{r}\right)+\right.$ c.c. $]$. Here the set of adjustable phases $\left(\varphi_{1}^{m}, \varphi_{2}{ }^{m}, \ldots, \varphi_{N}{ }^{m}\right)$ represents the address of the $m$ th image. In the readout cycle, the holograms will be recalled with these $N$ reference beams and the corresponding phase address. The amplitude of the resulting wave field that is incident upon the holographic medium during the recording of the $m$ th image is then given by

$$
\begin{aligned}
Q_{m}= & \frac{1}{2}\left[A_{m} \exp (j \mathbf{k} \times \mathbf{r})\right. \\
& \left.+\sum_{n=1}^{N} P_{n} \exp \left(j \mathbf{k}_{n} \times \mathbf{r}\right) \exp \left(j \varphi_{n}{ }^{m}\right)+\text { c.c. }\right] .
\end{aligned}
$$

If the recording medium is dynamic (e.g., a photorefractive crystal), then the beams are diffracted by the same index gratings they induce. This interaction always leads to energy or phase redistributions among the beams during their propagation inside the material. Because the successive recording of the images modifies previously recorded holograms, the relative phases and amplitudes of the reference beams are not the same during the readout and the recording processes. This phenomenon can be a source of cross talk between images. It depends on the recording material properties and on the recording scheme. For the following calculations, we consider the case in which the phases and amplitudes of the beams remain constant in the bulk of the holographic medium during the recording process. Therefore, from Eq. (1), we derive the intensity pattern inside the recording medium:

$$
\begin{aligned}
\left\|Q_{m}\right\|^{2}= & \frac{1}{2} A_{m} A_{m}{ }^{*}+\frac{1}{2} \sum_{n=1}^{N} P_{n} P_{n}{ }^{*} \\
& +\frac{1}{2} \sum_{n=1}^{N} A_{m} P_{n}^{*} \exp \left(-j \varphi_{n}{ }^{m}\right) \\
& \times \exp \left[j\left(\mathbf{k}-\mathbf{k}_{n}\right) \times \mathbf{r}\right] \\
& +\frac{1}{2} \sum_{n=1}^{N} P_{n} \sum_{l=1, l \neq n}^{N} P_{l}^{*} \exp \left[j\left(\varphi_{n}{ }^{m}-\varphi_{l}{ }^{m}\right)\right] \\
& \times \exp \left[j\left(\mathbf{k}_{n}-\mathbf{k}_{l}\right) \times \mathbf{r}\right]+\text { c.c. },
\end{aligned}
$$


where $l$ is an integer. For the sake of clarity, we have not included the scalar products of the polarization vectors in this equation.

Because phase and energy redistributions between the beams are neglected, the relative phases and amplitudes of the reference beams are the same for the recording and the readout processes. Therefore only the $N$ holograms induced by the interferences of the images with the $N$ reference beams are important for this readout process. Thus the resulting change in the refractive index that we have to take into account is

$$
\begin{aligned}
\Delta n_{m}= & \frac{1}{2\left(I_{m}+I_{R}\right)} \\
& \times\left\{\sum_{n=1}^{N} A_{m} P_{n}{ }^{*} \delta n_{n} \exp \left[-j\left(\varphi_{n}{ }^{m}+\psi\right)\right]\right. \\
& \left.\times \exp \left[j\left(\mathbf{k}-\mathbf{k}_{n}\right) \times \mathbf{r}\right]+\text { c.c. }\right\},
\end{aligned}
$$

where $\psi$ is the phase shift between the interference pattern and the refractive-index grating, $I_{m}=$ $0.5 A_{m} A_{m}{ }^{*}$ is the intensity of the image beam and $I_{R}=$ $0.5 \sum_{n=1}^{N} P_{n} P_{n}{ }^{*}$ is the total intensity of the reference beams.

$\delta n_{n}$ represents the amplitude of the index modulation of the grating whose wave vector is $\mathbf{k}-\mathbf{k}_{n}$. For instance, if the holographic medium is a photorefractive crystal ${ }^{22}$ we have

$$
\delta n_{n}=\beta \frac{n^{3} r_{\mathrm{eff}}^{n} E_{\mathrm{sc}}{ }^{n}}{2},
$$

where $n$ is the refractive index, $r_{\text {eff }}{ }^{n}$ is the effective electro-optic coefficient, $E_{\mathrm{sc}}{ }^{n}$ is the space-charge electric field and $\beta$ is a factor that depends on the recording procedure. It takes into account the erasure of previously recorded holograms by new ones.

With this method, all $M$ images can be superimposed and stored in the crystal. After recording, readout of the $p$ th image is performed with the $N$ reference beams illuminating the holographic medium with the corresponding phase address. To obtain an expression for the resulting diffracted amplitude $\mathscr{R}=0.5[R \exp (j \mathbf{k} \times \mathbf{r})+$ c.c. $]$, we use the coupled wave analysis. ${ }^{23}$ We assume that the light diffracted from the wave with wave vector $\mathbf{k}_{n}$ on gratings whose wave vectors are different from $\mathbf{k}_{n}$ $\mathbf{k}$, does not contribute to the output signal either because of Bragg selectivity or because of the spatial arrangement of the reference and signal waves. Therefore the first derivative equation of $R$ with respect to the space coordinate $z$ along the $\mathbf{k}$ vector is given by

$$
\begin{aligned}
\frac{\partial R}{\partial z}= & j \exp (j \psi) \frac{\pi}{\lambda} \sum_{m=1}^{M} \sum_{n=1}^{N} \delta n_{n} P_{n} \frac{A_{m} P_{n}{ }^{*}}{\left(I_{m}+I_{R}\right)} \\
& \times \exp \left[j\left(\varphi_{n}{ }^{p}-\varphi_{n}{ }^{m}\right)\right],
\end{aligned}
$$

where $\lambda$ is the wavelength of light. Once again, for the sake of clarity, we have not included in Eq. (5) the obliquity factors. ${ }^{23}$ This omission does not change our demonstration, and these coefficients can be included in the expression of $\delta n_{n}$, if needed.

In a real system, the index modulation $\delta n_{n}$ and the amplitude $P_{n}$ differ from one beam to another. As we show in Section 3, this can be a source of cross talk between images. However, in order to understand the principle mechanism of the phase-encoding technique, we now consider an ideal system in which $\delta n_{n}$ and $P_{n}$ do not depend on the beam we consider and are equal to $\delta n$ and $P_{0}$.

By setting $I_{0}=0.5 P_{0} P_{0}{ }^{*}$, we find that Eq. (5) simplifies to

$$
\begin{aligned}
\frac{\partial R}{\partial z}= & j \exp (j \psi) \frac{\pi}{\lambda} \delta n P_{0} \sum_{m=1}^{M} \frac{A_{m} P_{0}{ }^{*}}{\left(I_{m}+N I_{0}\right)} \\
& \times \sum_{n=1}^{N} \exp \left[j\left(\varphi_{n}{ }^{p}-\varphi_{n}{ }^{m}\right)\right] .
\end{aligned}
$$

Equation (6) demonstrates that the $p$ th image will be reconstructed with maximum diffraction efficiency if it is recorded with a modulation ratio of the interference pattern

$$
\frac{\sqrt{N} A_{m} P_{0}^{*}}{\left(I_{m}+N I_{0}\right)}
$$

equal to unity, that is, with the intensity $I_{m}$ equal to $N I_{0}$. However, we are interested in maximum diffracted power. Because we assume that the writing and readout intensities are the same, we must optimize the term

$$
\frac{N A_{m} P_{0}^{*} P_{0}}{\left(I_{m}+N I_{0}\right)}
$$

Therefore the maximum diffracted power is obtained for $I_{m}=N I_{0} / 2$.

Let us now consider the condition for which the $p$ th image $\left(R \equiv A_{p}\right)$ can be retrieved without cross talk. For that case, we have to address the system with phase codes that fulfill the conditions

$$
\begin{array}{ll}
\sum_{n=1}^{N} \exp \left[j\left(\varphi_{n}{ }^{p}-\varphi_{n}{ }^{m}\right)\right]=0 & \text { for } p \neq m, \\
\sum_{n=1}^{N} \exp \left[j\left(\varphi_{n}^{p}-\varphi_{n}{ }^{m}\right)\right]=N & \text { for } p=m
\end{array}
$$

Condition (7) is similar to problems appearing in matrix algebra. Therefore the problem can be reduced to find matrices $\left(X_{i j}\right)$ with

$$
\left(X_{i j}\right) \times\left(X_{j i}\right)^{*}=I,
$$

where $I$ is the unity matrix.

The solution of Eq. (8) is an unreducible basic matrix in which all column vectors of $\left(X_{i j}\right)$ are orthog- 
onal. As a result, we have to choose orthogonal phase codes to retrieve the $p$ th image without cross talk. For the special case of the binary phases that we are interested in (in which $\varphi_{n}{ }^{m}$ are equal to 0 or $\pi$ ), the vectors can be described as:

$$
\begin{array}{r}
{\left[\exp \left(j \varphi_{1}{ }^{m}\right), \exp \left(j \varphi_{2}{ }^{m}\right), \ldots, \exp \left(j \varphi_{N}{ }^{m}\right)\right]} \\
=( \pm 1, \pm 1, \ldots, \pm 1)=C_{m},
\end{array}
$$

Thus, for vectors of length $N$, a maximum of $N$ orthogonal phase addresses can be constructed if $N$ is a power of 2 . This construction may be obvious for a small number of phase codes, but when $N$ grows larger the Walsh-Hadamard transform ${ }^{24}$ may be used to construct the orthogonal set of phase addresses properly.

For $N=2$ this construction procedure leads to

$$
\left.\begin{array}{l}
C_{1}=(1,1) \\
C_{2}=(1,-1)
\end{array}\right\} \Rightarrow M_{2}=\left[\begin{array}{rr}
1 & 1 \\
1 & -1
\end{array}\right],
$$

and for $N=4$ from $N=2$ gives

$$
\begin{aligned}
& M_{4}= {\left[\begin{array}{ll}
M_{2} & M_{2} \\
M_{2} & -M_{2}
\end{array}\right]=\left(\begin{array}{rr}
1 & 1 \\
1 & -1
\end{array}\right]\left[\begin{array}{rr}
1 & 1 \\
1 & -1
\end{array}\right] } \\
& {\left.\left[\begin{array}{rr}
1 & 1 \\
1 & -1
\end{array}\right]-\left[\begin{array}{rr}
1 & 1 \\
1 & -1
\end{array}\right]\right) } \\
& \Rightarrow\left\{\begin{array}{l}
C_{1}{ }^{\prime}=(1,1,1,1) \\
C_{2}{ }^{\prime}=(1,-1,1,-1) \\
C_{3}{ }^{\prime}=(1,1,-1,-1) \\
C_{4}{ }^{\prime}=(1,-1,-1,1)
\end{array}\right.
\end{aligned}
$$

The successive application of this rule leads to arbitrary large orthogonal matrices.

The above calculations show that the maximum number $M$ of images that can be stored in the recording medium is equal to $N$. The theoretical image capacity of the phase-encoding technique is thus the same as for other angular multiplexing techniques. However, in the following we demonstrate that this image capacity is, in fact, limited by the imperfections of available optical components.

\section{Constraints of Real Systems}

Above we considered a perfect system [Eq. (6) and condition (7)]. However, when we switch to real systems, cross talk may arise as a limiting factor. For example, in a previous demonstration, 21 we observed that the temporal phase instabilities of the phase modulator (a commercial liquid-crystal television screen) greatly contributes to this noise. Hereafter we assume that these instabilities are absent (by using a binary ferroelectric liquid-crystal modulator, for instance) and we seek other possible origins of noise. From Eq. (5), we define the signal $S_{p}$ of the $p$ th image and its noise $B_{p}$ as follows:

$$
\frac{\partial S_{p}}{\partial z}=j \exp (j \psi) \frac{\pi}{\lambda} A_{p} \sum_{n=1}^{N} u_{n p},
$$

$$
\begin{aligned}
\frac{\partial B_{p}}{\partial z}= & j \exp (j \psi) \frac{\pi}{\lambda} \sum_{m=1, m \neq p}^{M} A_{m} \sum_{n=1}^{N} u_{n m} \\
& \times \exp \left[j\left(\varphi_{n}{ }^{p}-\varphi_{n}{ }^{m}\right)\right],
\end{aligned}
$$

with

$$
u_{n m}=\frac{P_{n} P_{n}{ }^{*}}{I_{m}+I_{R}} \delta n_{n} .
$$

The imperfections of the phase modulator $\varphi_{n}{ }^{p}-$ $\varphi_{n}{ }^{m} \neq \pi$ on the one hand and the variations in the diffracted amplitude (arising from $u_{n m}$ ) on the other hand, may give rise to a certain amount of crosscorrelation noise. These variations of $u_{n m}$ include both the nonuniformity in the refractive-index modulation and the differences between the referencebeam intensities. Below we derive a statistical analysis of these limitations in more details.

We consider the case in which each pixel of each image is encoded with binary information: the amplitude $A_{p}$ of these pixels is a random variable that takes the two values 0 and 1 with the same probability of $1 / 2$ (which corresponds to the greatest density of information).

\section{A. Limitations Imposed by Variations in the Diffracted Amplitude}

Here we consider a perfect phase-encoding element and we study the influence of spatial variations of the diffracted amplitude.

We can express the noise $B_{p}$ from Eq. (13):

$$
\frac{\partial B_{p}}{\partial z}=j \exp (j \psi) \frac{\pi}{\lambda} t_{p}
$$

with

$$
\begin{aligned}
t_{p} & =\sum_{m \neq p} w_{m}=\sum_{m \neq p} A_{m} v_{m} \\
& =\sum_{m \neq p} A_{m}\left(\sum_{\varphi_{n}^{m}-\varphi_{n}^{p}=0}\left|u_{n m}\right|-\sum_{\varphi_{n}^{m}-\varphi_{n}^{p}=\pi}\left|u_{n m}\right|\right) .
\end{aligned}
$$

We approximate $\left|u_{n m}\right|$ by a random variable with a mathematical expectation $\langle u\rangle$ and a variance $\sigma_{u}{ }^{2}$. The signal- $\left(A_{p}=1\right)$ to-noise ratio, defined in amplitude, is thus equal to

$$
r=\frac{N\langle u\rangle}{t_{p}} .
$$

Step by step we derive the mathematical expectations and variances of $v_{m}, w_{m}$, and $t_{p}$. Finally we find that

$$
\langle t\rangle=0, \quad \sigma_{t}^{2}=\frac{N(M-1)}{2} \sigma_{u}^{2} .
$$

Because $N$ and $M$ are much larger than unity, the probability law of $t_{p}$ can be approximated by a normal law. Therefore the probability of the signal-to-noise 
ratio $r$ being larger than $r_{0}$ is given by

$$
\operatorname{Prob}\left(|r|>r_{0}\right)=\frac{2}{\sqrt{2 \pi}} \int_{0}^{\left(r_{0 \sigma^{\prime}}\right)^{-1}} \exp \left(-t^{2} / 2\right) \mathrm{d} t
$$

with

$$
\sigma^{2}=\frac{(M-1)}{2 N} \frac{\sigma_{u}^{2}}{\langle u\rangle^{2}} .
$$

It is interesting to note that for maximum capacity $(M=N$ and $N \gg 1$ ) this probability does not depend on $N$. This probability can be made quite large. For instance, for maximum capacity $(N=M)$ and for fluctuations in the diffracted amplitude of $\sim 20 \%$ $\left(\sigma_{u} /\langle u\rangle=0.2\right)$ we find $\operatorname{Prob}(|r|>3)=0.98$. This probability decreases quickly as soon as the fluctuations in the diffracted amplitude becomes larger than $20 \%$. In order to reduce this noise, it is thus quite important to observe the two following requirements:

(a) The reference beam intensities must be equalized, (b) the fluctuations in the index modulation must be reduced. These fluctuations may originate first from the recording procedure, and second from the dependence of the photorefractive effect versus the holographic grating fringe spacing. It is thus more appropriate to use the incremental recording procedure ${ }^{20}$ and to make the reference beams angularly as close as possible.

We conclude that the fluctuations in the diffracted amplitude induce cross talk between images. This noise is inherent to the phase-encoding multiplexing while it is absent in other angular multiplexing techniques. However, this short study shows that this noise can be easily kept at a reasonably low level that is independent of the capacity $M$.

\section{B. Limitations Imposed by Imperfections of the Phase Modulator}

Now we assume that the diffracted amplitude is constant $\left(u_{n m}\right.$ is equal to $\left.u_{0}\right)$ but that the phase encoder suffers from imperfections. Then we can express the noise $B_{p}$ from Eq. (13):

$$
\frac{\partial B_{p}}{\partial z}=-\exp (j \psi) \frac{\pi}{\lambda} u_{0} t^{\prime}
$$

with

$$
t^{\prime}=-j \sum_{m \neq p} A_{m} \sum_{n=1}^{N} \exp \left[j\left(\varphi_{n}{ }^{p}-\varphi_{n}{ }^{m}\right)\right] .
$$

The signal-to-noise ratio $r$ is now defined as $r=N / t^{\prime}$.

We now study the influence of the imperfections of the phase encoder; $\varphi_{n}{ }^{p}-\varphi_{n}{ }^{m}=\pi+\epsilon$ instead of $\pi$, where $|\epsilon| \ll \pi$ is the phase noise. For sake of simplicity, we first examine the case in which $\epsilon$ is a random variable with a mathematical expectation equal to zero and a variance $\sigma_{\epsilon}^{2}$. In that case, we find that the mathematical expectation of $t^{\prime}$ is also zero and that its variance is

$$
\sigma_{t^{\prime}}{ }^{2}=\frac{\sigma_{\epsilon}^{2}(M-1) N}{2} .
$$

Because $M$ and $N$ are much larger than unity, $t^{\prime}$ obeys a normal law. The probability for the signal-tonoise ratio $r$ to be larger than $r_{0}$ is given by

$$
\operatorname{Prob}\left(|r|>r_{0}\right)=\frac{2}{\sqrt{2 \pi}} \int_{0}^{\left(r_{0} \sigma^{\prime \prime}\right)^{-1}} \exp \left(-t^{2} / 2\right) \mathrm{d} t,
$$

with

$$
\sigma^{\prime 2}=\frac{(M-1)}{2 N} \sigma_{\epsilon}^{2} .
$$

Once again, for maximum capacity, this probability does not depend on $N$. It is usually close to unity. For instance, for a system with $M-1 \approx N$ and for $\sigma_{\epsilon}=0.15$ we find $\operatorname{Prob}(|r|>3)=1-\left(2 \times 10^{-3}\right)$. We conclude that this random phase noise can be neglected and that is does not limit the capacity of the phase-encoding multiplexing technique.

Now we study the case of a systematic phase noise $\varphi_{n}{ }^{p}-\varphi_{n}{ }^{m}=\pi+\epsilon$, where $\epsilon$ is a constant. In this case $t^{\prime}$ is a random variable with variance

$$
\sigma_{t^{\prime}}{ }^{2}=(M-1) \frac{(N \epsilon)^{2}}{2}
$$

and with mathematical expectation

$$
\left\langle t^{\prime}\right\rangle=\frac{(M-1) N \epsilon}{2} .
$$

This mathematical expectation is quite large and may give rise to a large amount of noise. For instance, if we want the signal-to-noise ratio to be larger than $r_{0}$, then $\epsilon$ must fulfill the following inequality:

$$
\epsilon<\frac{2}{(M-1) r_{0}} .
$$

This inequality can be difficult to fulfill as soon as the image capacity becomes large.

In order to overcome this problem, we propose to add a supplementary spatially uniform phase shift on the image beam. At the beginning of the incremental recording, and for each image, a uniform phase shift is chosen at random between 0 and $\pi$. It remains the same for that image and for the following steps of the incremental procedure. Therefore, the mathematical expectation of $t^{\prime}$ becomes zero while its variance remains the same as before. Thus the probability for the signal-to-noise ratio $r$ to be larger than $r_{0}$ is now

$$
\operatorname{Prob}\left(|r|>r_{0}\right)=\frac{2}{\sqrt{2 \pi}} \int_{0}^{\left(r_{00}{ }^{\prime \prime}\right)^{-1}} \exp \left(-t^{2} / 2\right) \mathrm{d} t,
$$


with

$$
\sigma^{\prime \prime \prime 2}=\frac{(M-1)}{2} \epsilon^{2} .
$$

For instance, for $M=10^{3}$ and for $\epsilon=5 \times 10^{-3}$ we find that $\operatorname{Prob}(|r|>3)=1-\left(3 \times 10^{-3}\right)$.

From inequality (23) and Eq. (24) we observe that a systematic phase imperfection $\epsilon$ always induces a noise that increases with the image capacity $M$. This is the main limitation of the phase-encoding technique.

\section{Conclusion}

In conclusion we have investigated the advantages and limitations of a deterministic binary phaseencoding multiplexing technique. Compared with other angular multiplexing techniques, this method theoretically permits a high storage capacity without alignment problems. Moreover, easy, light efficient, and immediate image retrieval is possible. We have analyzed the different possible origins of cross talk between images and we have shown that the main limitation of the storage capacity $M$ of this technique arises from the imperfections of the phase modulator. In order to reduce that limitation, we proposed to add a supplementary uniform phase modulation on the image beam in such a way that the sum of all cross-correlation contributions during readout will destructively interfere and vanish. Capacities of approximately one thousand images seem to be realistic.

At the moment, experiments are in progress to implement a memory that will be used to feed an optical correlator with the stored images as filters.

\section{References}

1. P. J. Van Heerden, "Theory of optical information storage in solids," Appl. Opt. 2, 393-400 (1963).

2. D. Psaltis, D. Brady, and K. Wagner, "Adaptative optical networks using photorefractive crystals," Appl. Opt. 27, 17521759 (1988).

3. L. d'Auria, J. P. Huignard, C. Slezak, and E. Spitz, "Experimental holographic read-write memory using 3-D storage," Appl. Opt. 13, 808-818 (1974).

4. D. L. Staebler, W. J. Burke, W. Phillips, and J. J. Amodei, "Multiple storage and erasure of fixed holograms in Fe-doped $\mathrm{LiNbO}_{3}$," Appl. Phys. Lett. 26, 182-184 (1975).

5. H. Kurz, "Photorefractive recording dynamics and multiple storage of volume holograms in photorefractive $\mathrm{LiNbO}_{3}$," Opt. Acta 24, 463-473 (1977).
6. A. Marrakchi, W. M. Hubbard, S. F. Habiby, and J. S. Patel, "Dynamic holographic interconnects with analog weights in photorefractive crystals," Opt. Eng. 29, 215-224 (1990).

7. H. Lee, X. Gu, and D. Psaltis, "Volume holographic interconnections with maximal capacity and minimal cross talk," Appl. Opt. 65, 2191-2194 (1989).

8. D. Z. Anderson, "Coherent optical eigenstate memory," Opt. Lett. 11, 56-58 (1986).

9. A. Yariv and S.-K. Kwong, "Associative memories based on message-bearing optical modes in phase-conjugate resonators," Opt. Lett. 11, 186-188 (1986).

10. Y. Owechko, "Nonlinear holographic associative memories," Proc. IEEE 25, 619-634 (1989).

11. H. Xu, Y. Yuan, Y. Yu, K. Xu, and Y. Xu, "Performances of real time associative memory using a photorefractive crystal and liquid crystal electrooptic switches," Appl. Opt. 29, 3375-3379 (1990).

12. L. D'Auria, J. P. Huignard, and E. Spitz, "Holographic read-write memory and capacity enhancement by 3-D storage," IEEE Trans. Magn. MA-9, 83-94 (1973).

13. E. S. Maniloff and K. M. Johnson, "Dynamic holographic interconnects using static holograms," Opt. Eng. 29, 225-229 (1990).

14. J. E. Ford, Y. Fainman, and S. H. Lee, "Array interconnection by phase-coded optical correlation," Opt. Lett. 15, 1088-1090 (1990).

15. A. Marrakchi, "Continuous coherent erasure of dynamic holographic interconnects in photorefractive crystals," Opt. Lett. 14, 326-328 (1989).

16. J. T. LaMacchia and D. L. White, "Coded multiple exposure holograms," Appl. Opt. 7, 91-94 (1968).

17. P. Healey and D. Smith, "Holographic associative memory switching system," in Annual Meeting 1987, Vol. 22 of 1987 OSA Technical Digest Series (Optical Society of America, Washington, D.C., 1987), pp. 119-120.

18. T. F. Krile, M. O. Hagler, W. D. Redus, and J. F. Walkup, "Multiplex holography with chirp-modulated binary phasecoded reference-beam masks," Appl. Opt. 18, 52-56 (1979).

19. D. Z. Anderson and D. M. Lininger, "Dynamic optical interconnects: volume holograms as two-port operators," Appl. Opt. 26, 5031-5038 (1987).

20. Y. Taketomi, J. E. Ford, H. Sasaki, J. Ma, Y. Fainman, and S. H. Lee, "Incremental recording for photorefractive hologram multiplexing," Opt. Lett. 16, 1774-1776 (1991).

21. C. Denz, G. Pauliat, G. Roosen, and T. Tschudi, "Volume hologram multiplexing using a deterministic phase encoding method," Opt. Commun. 85, 171-176 (1991).

22. P. Günter and J. P. Huignard, eds., Photorefractive Materials and Their Applications I, Vol. 61 of Topics in Applied Physics (Springer-Verlag, Berlin, 1988), p. 26.

23. H. Kogelnik, "Coupled wave theory for thick hologram gratings," Bell Syst. Tech. J. 48, 2909-2947 (1969).

24. A. K. Jain, Fundamentals of Digital Image Processing (Prentice-Hall, Englewood Cliffs, N.J., 1988), p. 155. 